\title{
LASER SPECTROSCOPY OF ELECTRONIC RESONANCES: AN ELECTRO-OPTIC TECHNIQUE FOR PROBING COHERENT AND INCOHERENT DECAY PROCESSES*
}

\author{
A. H. Zewail \\ Arthur Amos Noyes Laboratory of Chemical Physics, ** \\ California Institute of Technology, Pasadena, California 91125
}

\begin{abstract}
A new technique is presented for measuring the incoherent resonance decay (IRD) and the coherent optical ringing of selectively ( $5 \mathrm{MHz}$ ) prepared electronic states. The method utilizes electro-optic switching of a single laser mode that is on or off resonance with respect to the homogeneous molecular packets in the excited ensemble. The technique was applied to a variety of systems (gases at low pressures, gases at relatively high pressures, and solids at low temperatures) to give information about their phase memory (optical $\mathrm{T}_{2}$ processes), radiative and radiationless decay (optical $\mathrm{T}_{1}$ processes), and to measure directly their optical transition moment between the ground state and the prepared electronic state. The theory of coherent and incoherent states is also given.
\end{abstract}

\section{Introduction}

Many years of theoretical and experimental work have been devoted to the understanding of the decay and preparation of electronic states in "isolated" molecules and in the condensed phase. The questions that have usually been asked focus on what happens to "primary" states following absorption from the photon field. Green's function techniques have been used (1) successfully to describe the coupling between discrete optical levels and continua of molecular vibrations, (1) lattice phonons, (2) and the radiative field of photon packets. (3) The latter explained theoretically the on-resonance and off-resonance effects of light pulses scattered by molecular states, as demonstrated by the beautiful experiments of Williams et al. (4) However, to understand fully the many interactions that lead into spectral broadening of the primary states, one is forced to answer the following questions:

1) What are the radiative and nonradiative decays (optical $T_{1}$ processes) that contribute to the width of the resonance?

2) Are excited electronic states optically coherent? In other words, what is the phase memory (optical $\mathrm{T}_{2}$ processes) of excited states in gases and solids?

3) How do optical $T_{1}$ and $T_{2}$ processes depend on the vibronic energy?

In this paper we show that both the optical coherent (forward) decay and the incoherent (right-angle) ${ }^{\dagger}$ decay of the prepared state can be monitored while the system is on and off resonance with a single mode ( $5 \mathrm{MHz}$ ) of a tunable dye laser. This new technique, which utilizes an electro-optic switching of a specific laser mode between different sub-ensembles of the excited system, is applied to a variety of systems (gases at low pressure, gases at high pressure, and solids at very low temperatures). We believe that much will be learned about the decay (like spontaneous emission, soft and hard collisions, phonon scattering in solids, and dipole dephasing) and the preparation of selective (within $5 \mathrm{MHz}$ ) states. The advantage of this technique relies on its simplicity. In contrast to pulsed lasers which span a larger frequency distribution, the utilization of a cw single mode dye laser stimulates specific molecular "packets" and provides a continuous tunable source for measuring small anisotropy in the decay channels. Moreover, where it is difficult to obtain coherent absorption, the incoherent resonance decay can be detected using the emission intensity which is very sensitive to the relaxation and the nature of the prepared state.

\section{What is Coherence?}

Coherence is a manifestation of the phenomenon of interference in quantum mechanics. In other words, if we have two electronic states (say, the ground state $\psi_{\mathrm{g}}$ and an excited electronic state $\psi_{\mathrm{e}}$ ) and we manage to form a superposition of these two states, a coherent $\mathrm{g}$ state will be formed:

$$
\psi(r, t)=C_{g} e^{-i \omega t} \psi_{g}(r)+C_{e} e^{-i \omega} e^{t} \psi_{e}(r)
$$

where $\mathrm{C}_{\mathrm{g}} \mathrm{C}_{\mathrm{g}}{ }^{*}$ is the probability of finding the system in the $\mathrm{g}$ state and $\omega_{\mathrm{g}}=\mathrm{E}_{\mathrm{g}} / \hbar$. This coherent state has
two

* This work was supported in part by the Sloan Fund.

** Contribution No. 5427 .

$\dagger$ By right-angle we mean the emission perpendicular to the propagation of the laser beam. 
(a) The density matrix for an ensemble of such levels is nondiagonal, i.e., a pure state is created with

$$
\underset{\approx}{\rho}=\left(\begin{array}{ll}
\mathrm{C}_{\mathrm{g}} \mathrm{C}_{\mathrm{g}}^{*} & \mathrm{C}_{\mathrm{g}} \mathrm{C}^{*} \mathrm{e}^{+\mathrm{i} \omega_{\mathrm{o}} \mathrm{t}} \\
\text { C.C. } & \mathrm{C}_{\mathrm{e}} \mathrm{C}^{*}
\end{array}\right)=\left(\begin{array}{cc}
\rho_{\mathrm{gg}} & \rho_{\mathrm{ge}} \\
\rho_{\mathrm{eg}} & \rho_{\mathrm{ee}}
\end{array}\right)
$$

where $\omega_{0}$ is the resonance frequency and C.C. denotes the complex conjugate.

(b) The expectation value for the moment $\mu$ is oscillating in time. Assuming that the ground and excited states have no permanent dipole moment,

$$
\langle\mu\rangle=\mathrm{C}_{\mathrm{g}} \mathrm{C}^{*} \mu_{\mathrm{ge}} \mathrm{e}^{\mathrm{i} \omega_{0} \mathrm{t}}+\text { C.C. }
$$

This quantum mechanical dipole moment radiates light in the same way as a classical oscillating "charge on a spring".(5) Indeed, this simple description explains the many interesting and exciting experiments (such as photon echoes) done recently in atomic, ionic, and molecular systems.

We notice that if the excited ensemble can be described by the above pure density matrix, the system is coherent for the entire lifetime of the state. On the other hand, if the ensemble is "impure", a phase shift will exist among the sub-ensembles (i.e., the different two-levels) such that coherence will be lost. Naturally the intermediate limit is challenging for reasons which raise the following two questions: (i) how can one measure the degree of coherence in mixed states and (ii) what causes the creation of the impure (partially coherent or completely incoherent) state?

\section{Two-Level System in a Laser Field}

The treatment of the interaction between a pulse of radiation (width $t_{p}<T_{1}, T_{2}$ ) and a two-level system is well-known since 1946 when Bloch (6) predicted the existence of phase $p$ coherence in a spin ensemble and Hahn (7) in 1950 demonstrated in a classical experiment the dephasing and refocusing of the ensemble moments as a result of finite coherence time that is longer than Rabi's oscillation time in the rotating frame. Hahn's discovery showed clearly that the reversibility of free induction decay exists. The extension of such ideas to the optical region was shown by Kurnit et al.(8) In their experiments an echo resulted from the in-plane refocusing of the homogeneous optical moments. This discovery has triggered similar experiments (9) done on other systems. Tang and Statz (10) were the first to argue that a stepfunction light pulse in an amplifying medium would cause the system to nutate at a time determined by the leading edge of the pulse, and to decay with the relaxation time constants. Hocker and Tang (10) observed an optical nutation in $\mathrm{SF}_{6}$ gas when a pulse from a $10.6 \mu \mathrm{CO}$ laser was passed through the cell. Although the modulation was not obviously sinusoidal, it is fair to say that the power level they used in their experiments was in agreement with the theoretical prediction, and that they were the first to point out such an effect which has been observed clearly in the elegant experiments of Brewer and his co-workers.(11)

As we shall see, in our experiments both the diagonal and off-diagonal elements of the density matrix $(\rho)$ are being monitored in an optically thin sample. Being optically thin ensures that there are no propagation effects, such as self-induced transparency.(12) The sample emitting cooperatively resembles Dicke's superradiance or, more specifically, the linear combination (molecules plus field) of Dicke's states.(13) Therefore, the coupling between Maxwell's equations of the radiation field and the Schrödinger representation (14) of the two-level system becomes straightforward.

Defining the total decay time constant for the two levels as $\mathrm{T}_{1 \mathrm{e}}$ and $\mathrm{T}_{1 \mathrm{~g}}$, one can show that the rate of change of the elements of the density matrix is given by

$$
\begin{gathered}
-\dot{\rho}_{\mathrm{ee}}=\frac{\rho_{\mathrm{ee}}}{\mathrm{T}_{1 \mathrm{e}}}-\rho \\
-\dot{\rho}_{\mathrm{gg}}=\frac{\rho_{\mathrm{gg}}}{\mathrm{T}_{\mathrm{lg}}}+\rho \\
-\dot{\rho}_{\mathrm{eg}}=\left\{\mathrm{i}\left[\omega_{\mathrm{o}}+\delta(\mathrm{t})\right]+\left[\frac{1}{2}\left(\mathrm{~T}_{1 \mathrm{e}}^{-1}+\mathrm{T}_{1 \mathrm{~g}}^{-1}\right)\right]\right\} \quad \rho_{\mathrm{eg}}-\mathrm{i} / \hbar \mathrm{V}_{\mathrm{eg}}\left(\rho_{\mathrm{ee}}-\rho_{\mathrm{gg}}\right)
\end{gathered}
$$




$$
-\rho=i / \hbar v_{\text {eg }} \rho_{\text {ge }}+\text { c.c. }
$$

The above equations imply first that there exist differences in the decay if one monitors the diagonal and/or off-diagonal elements, i.e., the polarization component or the population difference between the two levels $\left(\rho_{e e}-\rho_{\text {gg }}\right)$. The latter is, of course, proportional to $\mathrm{R}_{3}$ in the FVH picture. (14) Second, the dipole which can be added to the $1 / \mathrm{T}_{1}$ term. This is true if one assumes a simple correlation function for the dephasing process in the gaseous or the solid ensemble when the interaction $V\left(V=-\mu \cdot E_{0} \cos \omega t\right)$ couples the radiation field (of frequency $\omega$ ) with the system of moment $\mu$. In our case, the laser field in the optical region couples with the electric dipoles that are generated from the transition between the ground state and a "narrow" optical electronic state. It is this coupling that creates the in-phase and the quadrature components of the polarization which evolve in time as follows:

$$
\begin{gathered}
\dot{\mathrm{R}}_{1}=-\Delta \mathrm{R}_{2}-\gamma \mathrm{R}_{1} \\
\dot{\mathrm{R}}_{2}=\Delta \mathrm{R}_{1}-\gamma \mathrm{R}_{2}+\left(\frac{\mu \mathrm{E}_{0}}{\hbar}\right) \mathrm{R}_{3} \\
\dot{\mathrm{R}}_{3}=\left(-\gamma \rho_{\mathrm{ee}}+\gamma_{\mathrm{g}} \rho_{\mathrm{gg}}\right)-\left(\frac{\mu \mathrm{E}_{0}}{\hbar}\right) \mathrm{R}_{2} .
\end{gathered}
$$

$\gamma_{\mathrm{e}}$ and $\gamma_{\mathrm{g}}$ are the decay rate constants of levels e and g, respectively, while $\gamma=\frac{\gamma_{\mathrm{e}}+\gamma_{\mathrm{g}}}{2}+1 / \mathrm{T}_{2} . \Delta$ is the frequency of the primary state in the "optical" rotating frame. $R_{1}$ and $R_{2}$ are directly related to $\rho_{\text {eg }}$ and $\rho_{\mathrm{ge}}$. The above Bloch-type equations imply that a macroscopic polarization $\mathrm{P}$ reacts back on the ${ }_{\text {ge }}$ applied field and causes a dispersion or absorption (or gain) depending on the relative phase between $P$ and the applied field. Moreover, the equations are reminiscent of the torque equation for the spin $\frac{1}{2}$ problem since

$$
\frac{d \vec{P}}{d t}=K\left(\vec{P} \times \vec{E}_{\text {eff }}\right)
$$

$\mathrm{K}$ is related to the dipole matrix element and $\overrightarrow{\mathrm{E}}_{\text {eff }}$ is the effective field. In the rotating frame and on-resonance, the population vector will be driven eff (nutation) in a plane perpendicular to the $\mu \mathrm{E}_{0}$ axis, depending on the strength of the moment and the laser field. Of course, when $E_{0}$ is switched off, $R_{3}$ will decay by its usual decay time constant. Hence, both $T_{1}$ and $T_{2}$ optical processes can be obtained in a solid or a gas when certain sub-ensembles are switched on and off resonance.

\section{The Incoherent Resonance Decay (IRD) Experiments}

In the IRD experiments, an intense single mode tunable dye laser was used for the excitation. $\mathrm{An}^{+} \mathrm{Ar}^{+}$ ion laser (Spectra Physics Model 170; total power = 18 watts) was used to pump a free jet stream dye laser. The dye solution was rhodamine 6G in ethylene glycol. A modified Spectra Physics (Model 580 A) dye laser was used so a single mode could be switched out of the transition resonance frequency by an electro-optic element. Special attention was given to the alignment procedure so the dispersion of the crystal in the electric field was caused by the modulation of the refractive index along the principal axes of the dielectric ellipsoid. The modulation is done transversely and is different from the switching procedure of Telle and Tang (15) used for very rapid tuning of $\mathrm{cw}$ dye lasers. The switching is clearly demonstrated in our experiments as shown in Figure 1. From the dispersion frequency we obtained an electro-optic dispersion for the $\mathrm{AD}^{*} \mathrm{P}$ crystal of $1.4 \mathrm{MHz} / \mathrm{V}$. The net laser power of the single mode can be varied up to $90 \mathrm{~mW}$ and the field is linearly polarized.

The single mode beam was split so both the frequency of the transition and the quality of the single mode can be checked during the experiments (see Figure 1). The single-mode spectrum was monitored with a Fabry-Perot interferometer that is scanned to allow the observation of on-resonance and off-resonance mode structure of the laser beam. Most of the laser beam intensity was used in the forward direction for the excitation of the sample. For the study of low pressure gases $\left(\mathrm{I}_{2}\right.$ and $\left.\mathrm{NO}_{2}\right)$, a sample tube with optical windows was used. A cold finger at the side of the tube was used to control the pressure of the gas. For solid state experiments, the crystal was oriented in a liquid helium Dewar that can be pumped to temperatures below the $\lambda$-point of liquid helium.

The emission was collimated at a right angle to the exciting beam with lenses of proper $f$ numbers, and focused on a cooled photomultiplier (EMI-9558). The photomultiplier was magnetically and electrostatically shielded. Since noise will be introduced because of the laser light scattering, a sharp cutoff was placed in 
front of the phototube. The output of the photomultiplier was terminated in a boxcar integrator (PAR Model 162) whose gate width was adjusted between $5-50$ nsec. The photomultiplier was coupled AC into the boxcar and the output was traced on an $\mathrm{X}-\mathrm{Y}$ recorder. Typical results are shown in Figure 2.

\section{IRD and COR Experimental Setup}
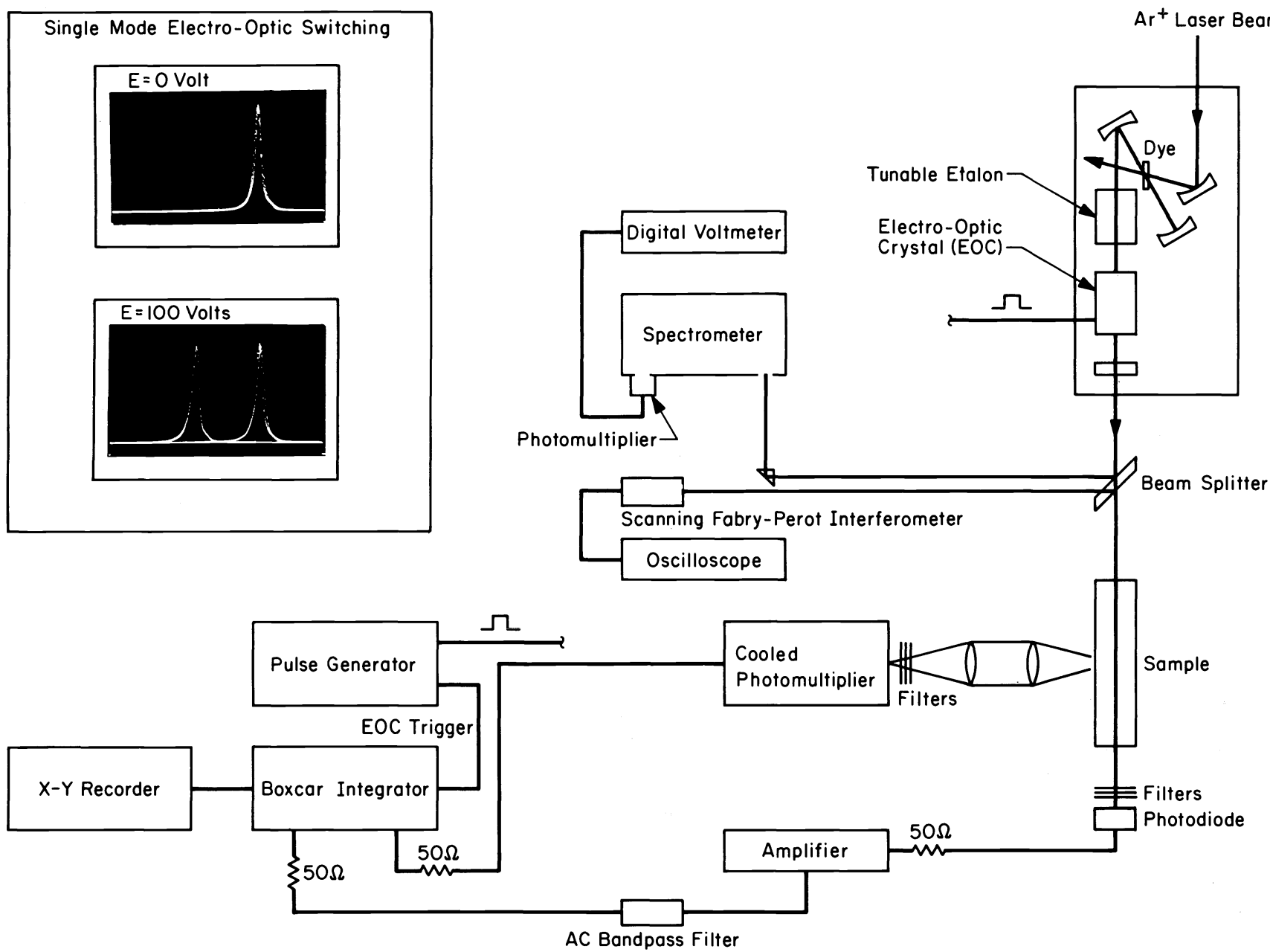

Fig. 1. A schematic diagram of the experimental setup for the incoherent resonance decay (IRD) and coherent optical resonance (COR) experiments. The insert in the figure shows the single mode of the $\mathrm{cw}$ dye laser when the voltage on the electro-optic crystal $(1.4 \mathrm{MHz} / \mathrm{V})$ is zero and $100 \mathrm{~V}$. The mode spectrum was monitored using an interferometer (see text). To minimize the jitter of the mode, the optics of the laser were carefully mounted on an NRC vibration-isolation table. 


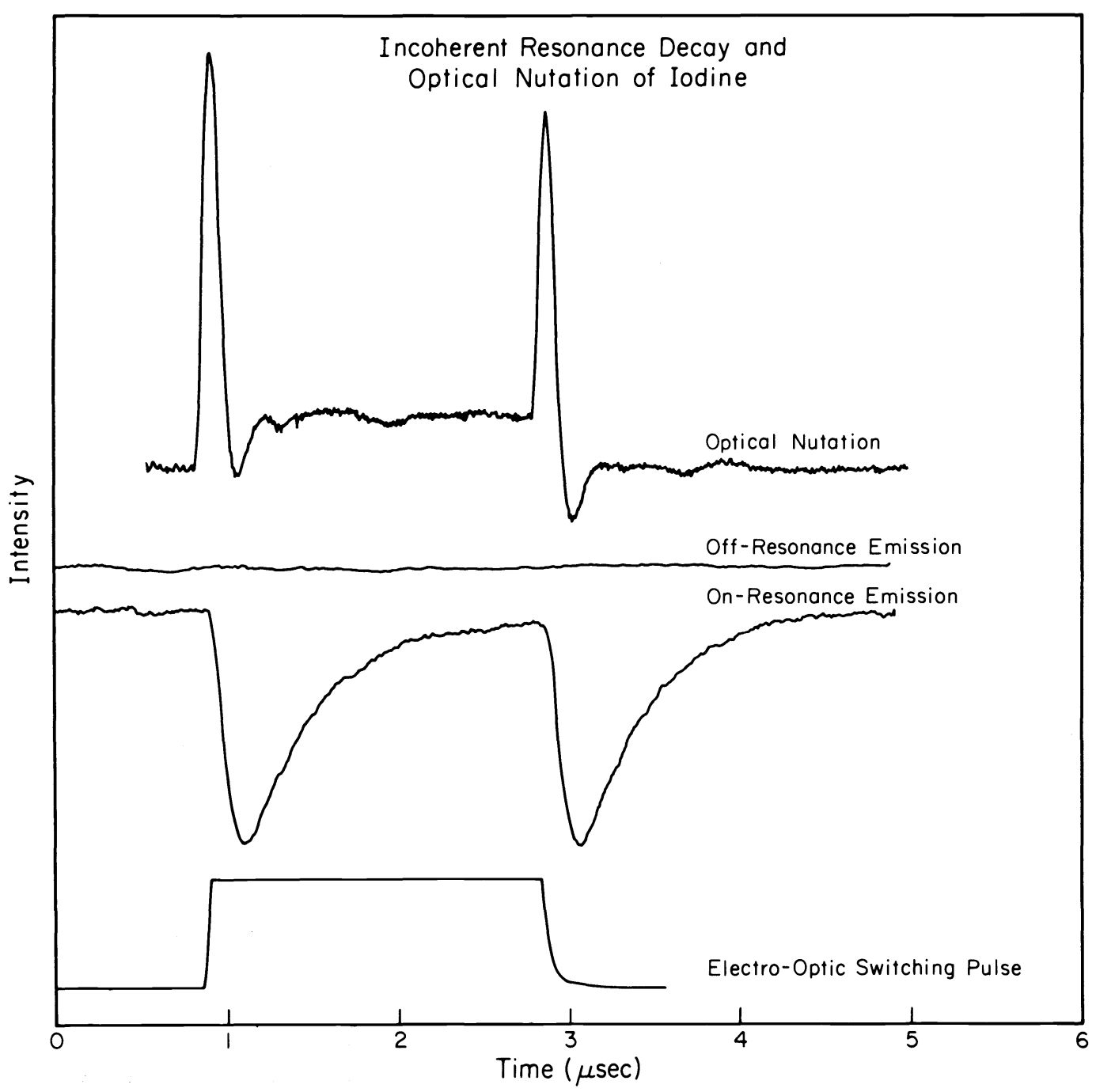

Fig. 2. The boxcar output plots of the on-resonance and off-resonance em ission decay when the electrooptic switching pulse is on and off. Also shown in this figure is the optical nutation of iodine at $0^{\circ} \mathrm{C}$. The on-resonance emission was taken at $14 \mathrm{MHz}$ switching frequency while the nutation was taken at $4 \mathrm{MHz}$.

\section{The Coherent Optical Resonance (COR) Experiments}

The experimental setup for COR is identical to IRD except we monitored the laser intensity in the forward direction. Carefully mounted photodiodes were used in combination with filters to obtain the transients. These transients will appear either as coherent absorption and emission, as in the optical nutation (see Figure 2), or as beating with the incident beam, as in the free induction decay (Figure 3) signal.(16) The output of the biased diode (with constant voltage supply) was amplified and fed into either an oscilloscope of the boxcar integrator. The laser power was measured by a Spectra Physics power meter (Model 401B). Knowing the power of the single mode and the beam diameter gave the radiation density at the sample. The coherent transients in the gas phase are very sensitive to the pressure in the cell while the coherent transients of the solid depend crucially on the crystal temperature, as evident from our work on pentacene in P-terphenyl between $1.8^{\circ} \mathrm{K}$ and above the $\lambda$-point of liquid helium. 


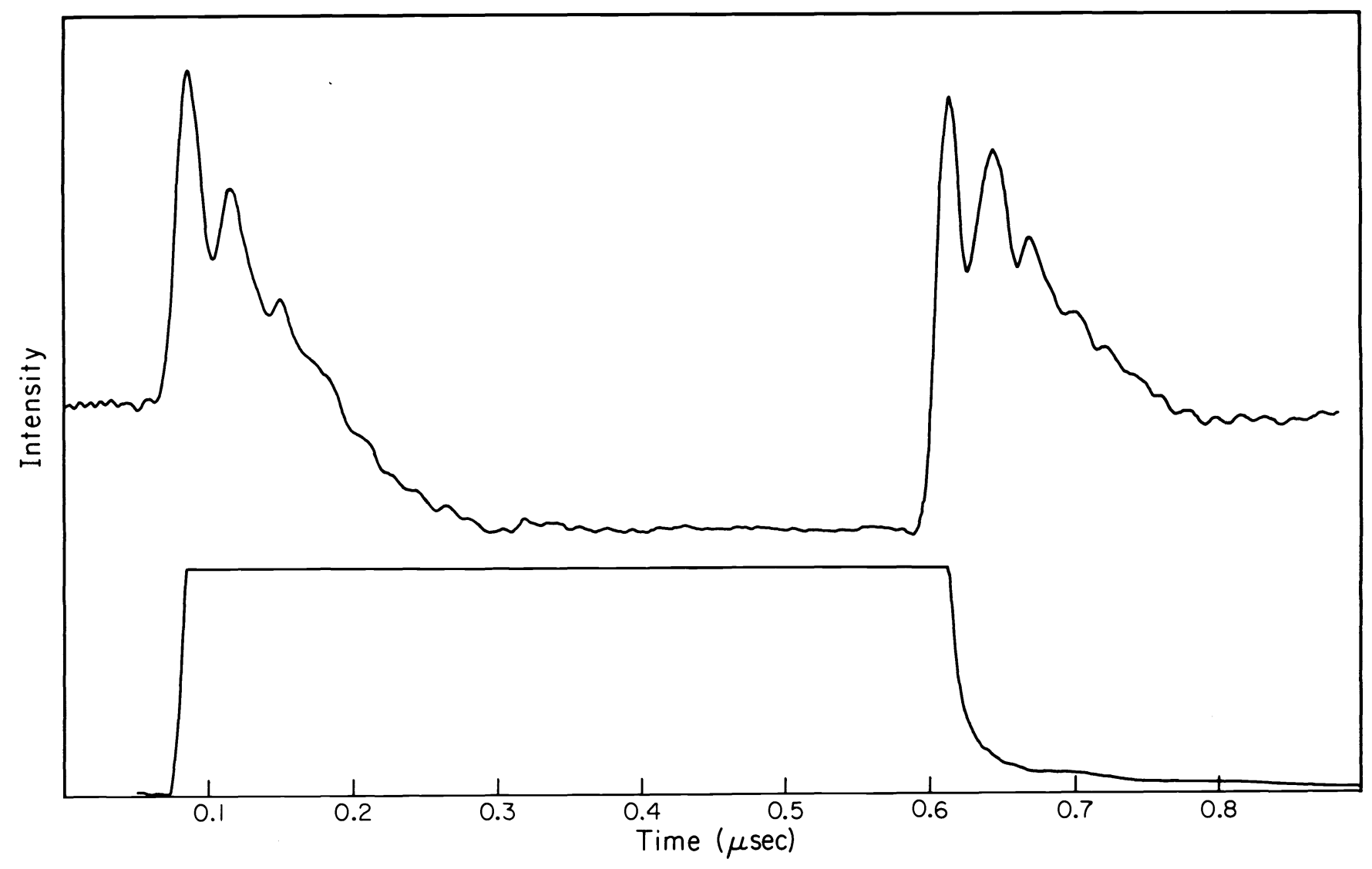

Fig. 3. The free induction decay of iodine gas with the cold finger at $0^{\circ} \mathrm{C}$ (switching frequency is $38 \mathrm{MHz}$ ) coinciding with the leading and trailing edges of the EOC pulse.

\section{Optical $\mathrm{T}_{1}$ and $\mathrm{T}_{2}$ Processes of Excited States}

Using the above techniques in this series of experiments and in other experiments that will be published elsewhere, we are investigating in a systematic way the optical $\mathrm{T}_{1}$ and $\mathrm{T}_{2}$ processes. The small, intermediate, and large molecule limits, both a low pressures and in the solid phase, are examined. The objectives are to obtain information about the incoherent and coherent decays from this new technique and to correlate them with the findings of the many theories that already exist about the nature of primary excited states.

Population Distribution of the Inhomogeneous Gaseous Ensemble

We start with the prototype $I_{2}$ molecule where we expect a "clean" excitation distribution and minimum vibronic scrambling. Naturally we must consider the Doppler broadening of the transition resonances ( $\mathrm{T}_{2}^{*}$ ). Another important system, which certainly is not as simple electronically as iodine, is $\mathrm{NO}_{2}$ on which decades of work have already been spent to understand its spectroscopy.

The $\mathrm{I}_{2}$ gas was excited at 5897.5 $\AA$ and Figure 2 shows the decay of the iodine on-resonance and offresonance when the electro-optic switch is modulating the laser beam. The gas pressure was controlled by using different temperature baths at the cold finger. Typically, 30 mtorr was used. At this pressure both the coherent transients and IRD were monitored. We also checked the signal at liquid nitrogen temperature and at room temperature and no decay was observed.

Two sub-ensembles must be considered in treating the decay and dephasing of the dipoles in the inhomogeneous ensemble. The first group of molecules consists of those that were originally excited by the single mode $\mathrm{cw}$ laser. The degree of inversion after long times (compared with relaxation times) can be obtained from Eqs. (8) - (10). On-resonance and if the laser intensity is sufficiently large, $R_{3} \sim 0$. Turning the pulse on with $\Delta \gg 1 / T_{2}$, this group of molecules will decay freely while a new group of molecules having $R_{3}^{0}=-1$, $\mathbf{R}_{2}^{0}=\mathbf{R}_{1}^{0}=0$ will be coherently driven. Hence, one expects the emission intensity, which monitors the diagonal elements of the density matrix, to build up and decay. 
In the iodine case, the IRD fast decay constant was found to be $0.23 \mu \mathrm{sec}$ at $105 \mathrm{mtorr}$. Stern and Volmer (17) have shown that the total decay may be partitioned into a radiative part and another part which measures the number of deactivating collisions. The latter depends on the pressure of the gas and the molecular collision diameter $\sigma$. Using our results we obtain a radiative decay constant of $1.3 \mu \mathrm{sec}^{-1}$ which is in excellent agreement with the results of phase fluorometry (18) done on $\mathrm{I}_{2}$ gas. By lowering the gas pressure (almost a collisionless gas), the decay time constant gets longer as expected from simple radiationless decay theory. Currently we are investigating the nature of the decay as a function of pressure and laser frequency. This will enable us to establish the relationship between the effective density of states (19) and the excess rovibronic energy in intervals of $10^{-4}$ wavenumbers. Hence, the "sparse" intermediate limit (20) (as opposed to the statistical or resonance limits) for the coupling between the prepared state and the rovibronic continua can be clearly identified. The beating between the different levels of the system during the decay can also be seen since our switching procedure is, perhaps, fast enough to give the appropriate time resolution. As mentioned before, the IRD experiments were also tried at $77^{\circ} \mathrm{K}$ and at room temperature (where a very strong emission was seen) and no signal was detected.

For $\mathrm{NO}_{2}$ the situation is different from iodine. The density of vibrational ground states that are quasiresonant with the excited level is higher. Moreover, these nonlinear molecules undergo relatively large geometrical changes when excited. This results in a large vibrational Franck-Condon overlap factor which makes the vibronic coupling matrix elements rather large and hence a short decay time is expected. At room temperature the Doppler width of $\mathrm{NO}_{2}$ near $4880 \mathrm{~A}$ is $\sim 1 \mathrm{GHz}$. In our experiments the laser excites $\mathrm{NO}_{2}$ at $5935.7 \AA$ where the absorption and fluorescence $(0.1 \mathrm{mtorr})$ at low resolution is known. (21) The coherent decay at relatively high pressure is very fast ( $\leqslant 65 \mathrm{nsec})$ and again disappears at low temperatures. We hope to report soon on the complete study of $\mathrm{NO}_{2}$ decay characteristics at different pressures and excitation energies.

\section{Coherent Pumping and the Optical Transition Moment}

As we described before, in the rotating frame and on-resonance the macroscopic moment [see Eqs. (8)-(11)] will nutate optically at Rabi's frequency. (22) Hence, if we know the transition rate for the coherent pumping and the electric field strength at the sample, we can compute the transition moment quite easily:

$$
\left(\mu \cdot E_{0} / \hbar\right)=\text { oscillation frequency. }
$$

Knowing the laser intensity (after the first glass window of the cell) and measuring the width of the beam profile (FWHM) at the iodine cell gave $\mathrm{E}_{0}=105 \mathrm{~V} / \mathrm{cm}$. These preliminary results therefore give $\mu=0.05$ Debye for the resonance transition. To our knowledge, this is the first time that this quantity has been measured directly for an electronic transition. (23)

The coherent pumping rate on-resonance can also be used to obtain information about the dipole dephasing processes. From the results of iodine at relatively low pressure (gas temperature is near $0^{\circ} \mathrm{C}$ ),
we obtained a pumping rate of $2.1 \times 10^{6} \mathrm{c} / \mathrm{sec}$ for the molecular packets that are driven on resonance. This value gave a $\mathrm{T}_{2}$ that predicts a free induction decay time constant of $\approx 50$ nsec since

$$
\frac{1}{\tau}=\mathrm{T}_{2}^{-1}+\left[\mathrm{T}_{1} \mathrm{~T}_{2}^{-1}\left(\frac{\mu \cdot \mathrm{E}_{0}}{\hbar}\right)^{2}+\mathrm{T}_{2}^{-2}\right]^{\frac{1}{2}}
$$

This is indeed in good agreement with our results (see Figure 3), thus ensuring the self-consistency and showing that this simple technique can be used for examining optical phase coherence and incoherent decay processes in excited states.

\section{The Phenomenon of Optical Coherence in Molecular Solids}

Using the above techniques we have observed for the first time the coherent optical ringing of molecular crystals in their excited states. Several systems (ionic and molecular) are being investigated in our laboratory and we present here the data on pentacene in p-terphenyl at $1.8^{\circ} \mathrm{K}$.

Pentacene in p-terphenyl is known to show four distinct sites in the absorption and emission, with a linewidth of $1-3 \mathrm{~cm}^{-1}$ at $1.8^{\circ} \mathrm{K}$. (24) These origins fluoresce when the laser is on-resonance. The socalled $\mathrm{O}_{1}$ origin was excited with a single mode of the dye laser and fluorescence was seen while monitoring the coherent transients of the $O_{1}$ resonance. When the electro-optic crystal was triggered so the laser was switched out of a particular "homogeneous molecular packet," we observed the transients shown in Figure 4. These signals were absent when the laser beam was blocked before the crystal and also when the crystal was out of the optical path. These fast transients, together with the IRD signal, disappeared after the superfluid helium was gone. This is expected since the optical spectral diffusion rate will increase when the phonon population increases. Of course this is being studied now in much more detail.

The fluorescence signal detected at a right angle follows the principles discussed before the incoherent resonance decay observed (25) in gases. In the solid the IRD pattern depends on the laser power and $\Delta$. 


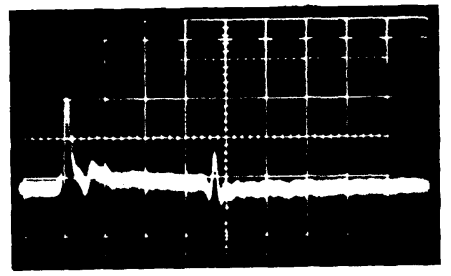

Fig. 4. Coherent optical resonance from solid pentacene in $\mathrm{p}$-terphenyl at $1.8^{\circ} \mathrm{K}$. This figure shows the COR signal (100 nsec/division) and

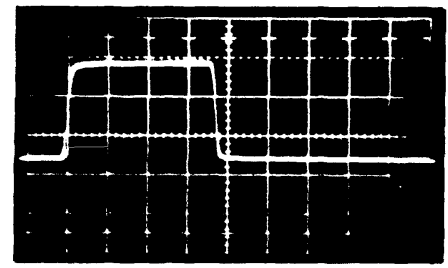
the electro-optic switching pulse.

From these preliminary results we estimate the optical coherence time to be $\sim 60$ nsec. This implies that dephasing of molecular dipoles in the solid occurs before emission into the ground state, even at $1.8^{\circ} \mathrm{K}$. We should add that the forward coherent signal was not observed when the laser mode was on-resonance with the vibronic origin near 5829 A. This observation (26) is in agreement with the conclusion that a fast decay process does exist (24a) in the vibrationally excited states of the solid. The details of this work are forthcoming.

\section{Acknowledgments}

I wish to thank Drs. J. Morris Weinberg and T. Hirschfeld for inviting me to present this work at the 20th Annual Symposium on Optical, Electro-optical, Laser and Photographic Technology (sponsored by the Society of Photo-Optical Instrumentation Engineers, SPIE) in August of 1976. I should point out that it was the work of T. E. Orlowski and D. R. Dawson of this laboratory which raised the issues discussed in this paper. I would also like to thank Kevin Jones for many hours of stimulating discussions.

\section{References}

1. R. A. Harris, J. Chem. Phys., 39, 978 (1963); C. A. Langhoff and G. W. Robinson, Mol. Phys., 26, 249 (1973); A. Nitzan, J. Jortner and P. M. Rentzepis, ibid., 26, 281 (1973).

2. A. Nitzan and J. Jortner, Mol. Phys., 25, 713 (1973); A. Nitzan, S. Mukamel and J. Jortner, J. Chem. Phys., 60, 3921 (1974), and references cited therein.

3. J. O. Berg, C. A. Langhoff and G. W. Robinson, Chem. Phys. Lett., 29,305 (1974); J. M. Friedman and R. M. Hochstrasser, Chem. Phys., 6, 155 (1974); S. Mukamel añd J. Jortner, J. Chem. Phys., 62, 3609 (1975).

4.' ج̂.' F. Williams, D. L. Rousseau and S. H. Dworetsky, Phys. Rev. Lett., 32,196 (1974).

5. R. Feynman, R. Leighton, and M. Sands, The Feynman Lectures on Physic $\widetilde{S}$ (Addison-Wesley Publishing Co., New York, 1964).

6. F. Bloch, Phys. Rev., 70, 460 (1946).

7. E. L. Hahn, Phys. Rev., 77, 297 (1950).

8. N. A. Kurnit, I. D. Abella and S. R. Hartmann, Phys. Rev. Lett., 13,567 (1964).

9. N. Takeuchi and A. Szabo, Phys. Lett., 50A, 361(1974); T. Aartsmã and D. A. Wiersma, Phys. Rev. Lett. , 36, 1360 (1976).

10. C. L. Tang and H. Statz, Appl. Phys. Lett., 10, 145 (1968); G. B. Hocker and C. L. Tang, Phys. Rev. Lett., 21, 591 (1969).

11. R. G. Brewer and R. L. Shoemaker, Phys. Rev. A, 6, 2001 (1972); R. Brewer and A. Genack, Phys. Rev. Lett., 36, 959 (1976).

12. S. L. McCâlf and E. L. Hahn, Phys. Rev. Lett. , 18, 908 (1967).

13. R. H. Dicke, Phys. Rev., 93, 99(1954).

14. R. P. Feynman, F. L. Verñon, Jr., and R. W. Hellworth, J. Appl. Phys., 28,49 (1957).

15. J. Telle and C. L. Tang, Appl. Phys. Lett., 24, 85 (1974); ibid., 26, 572 (1975).

16. R. Brewer and A. Genack (ref. 11) have reportêd recently the forward coherent transients in iodine. Our results on the coherent decay are in agreement with theirs. We should add that both Dr. Brewer's group and our group are trying the forward transient coherent experiments on different systems at the same time. So it is fair to say that there is and will be considerable activity in this field. 
17. O. Stern and M. Volmer, Z. Physik, 20, 183 (1919).

18. L. Brewer, R. Berg and G. Rosenblatt, J. Chem. Phys., 38, 1381 (1963).

19. The effective density of states here means the product of the actual density of states times the offdiagonal matrix element that connects the primary Born-Oppenheimer state with the continuum.

20. (a) G. W. Robinson and R. P. Frosch, J. Chem. Phys., 38, 1187 (1963); M. Bixon and J. Jortner, ibid., 48, 715 (1968); D. Chock, J. Jortner and S. A. Rice, ibid. ,49, 610 (1968); (b) For excellent review $\widetilde{s}$ by S. A. Rice and G. W. Robinson on the subject, see Excifed States, edited by E. C. Lim (Academic Press, New York, 1974 and 1975), Vols. I and $\Pi$.

21. C. G. Stevens, M. W. Swagel, R. Wallace and R. N. Zare, Chem. Phys. Lett., 18, 465 (1973).

22. R. L. Shoemaker and E. W. van Stryland, J. Chem. Phys., 64, 1733 (1976).

23. We are now making a complete fit for the coherent decay curve so an accurate measurement of the dipole moment can be obtained.

24. (a) A. P. Marchetti, W. C. McColgin, and J. H. Eberly, Phys. Rev. Lett., 35, 387 (1975); (b) J. H. Meyling and D. A. Wiersma, Chem. Phys. Lett., 20, $383(197 \overline{3})$.

25. A. H. Zewail, T. E. Orlowski, and D. R. Dawson, Chem. Phys. Lett., in press.

26. When the laser was near the 5829 A transition, the crystal was fluorescing, but not as strongly as when the laser was near the $\mathrm{O}_{1}$ origin at $5921.7 \AA$.

Question by John W. Daily, Univ. of Cal., Berkeley:
Answer: Yave you observed the solid spectrum at $90^{\circ}$ ?

Question: Do you measure a short decay period associated with the coherent portion of the $90^{\circ}$ spectrum?

Answer: Yes.

Question by C. Y. Robert Wu, Univ. of Iowa: Have you studied the temperature dependence of the phase relaxation time, say, particularly temperatures below $1.3^{\circ} \mathrm{K}$ ?

Answer: It is nontrivial to do the experiments below $1.3^{\circ} \mathrm{K}$; however we have studied the temperature dependence above $1.3^{\circ} \mathrm{K}$. As the temperature got higher and higher, the signal eventually disappeared.

Comment: When the temperature goes lower than $1.3^{\circ} \mathrm{K}$, the phase relaxation can be possibly as slow as the energy relaxation. In other words, as the temperature becomes lower than certain values, the phase relaxation time will approach the radiative lifetime. Therefore it is no longer temperature-dependent.

Reply: Perhaps this is true; however, the origin of dephasing is not really fully understood and we cannot just extrapolate to the very low temperature regions.

Question by Jerry Gelbwachs, The Aerospace Corp.: This is a very interesting technique. What kind of solids did you study?

Answer: We have studied both molecular and ionic crystals where the excitation is either localized at a certain site or delocalized over many sites (excitonic effects). 